\title{
A computational basis for higher-dimensional computational geometry and applications ${ }^{\text {th }}$
}

\author{
K. Mehlhorn ${ }^{\mathrm{a}, *}$, M. Müller ${ }^{\mathrm{b}}$, S. Näher ${ }^{\mathrm{c}}$, S. Schirra ${ }^{\mathrm{a}}$, M. Seel ${ }^{\mathrm{a}}$, C. Uhrig ${ }^{\mathrm{a}}$, J. Ziegler ${ }^{\mathrm{a}}$ \\ a Max-Planck-Institut für Informatik, Im Stadtwald, 66123 Saarbrücken, Germany \\ ${ }^{\mathrm{b}}$ RIB Bausoftware GmbH, Vaihinger Str. 151, 70507 Stuttgart, Germany \\ ${ }^{c}$ Fachbereich Mathematik und Informatik, Martin-Luther Universität Halle-Wittenberg, 06120 Halle, Germany
}

Communicated by C.M. Hoffmann; submitted 15 August 1997; accepted 6 January 1998

\begin{abstract}
In this paper we describe and discuss a kernel for higher-dimensional computational geometry and we present its application in the calculation of convex hulls and Delaunay triangulations. The kernel is available in form of a software library module programmed in $\mathrm{C}++$ extending LEDA. We introduce the basic data types like points, vectors, directions, hyperplanes, segments, rays, lines, spheres, affine transformations, and operations connecting these types. The description consists of a motivation for the basic class layout as well as topics like layered software design, runtime correctness via checking routines and documentation issues. Finally we shortly describe the usage of the kernel in the application domain. (C) 1998 Elsevier Science B.V.
\end{abstract}

Keywords: Software library; Implementation; Convex hull; Delaunay triangulation

\section{Introduction}

A growing community within computer science, academia and industry tries to transfer the theoretical algorithmic knowledge to practical usable programs. What already happened in other parts of computer science, namely the development of software libraries to speed up program implementation is now also an issue within geometric computing. We are now in a situation where the available computing power, the recent developments concerning exact arithmetic packages, and the identification of reasonable geometric primitives allow us to design a programming toolbox for this purpose.

We describe and discuss a kernel for higher-dimensional computational geometry. We have implemented all basic data types like points, vectors, directions, hyperplanes, segments, rays, lines, spheres, affine transformations, and operations connecting these types in form of C++ class objects. The kernel is structured into three layers:

\footnotetext{
This work was supported by ESPRIT Project 21957 (CGAL).

* Corresponding author.
} 
- arbitrary precision integer and rational arithmetic (classes integer and rational),

- exact linear algebra (classes integer_vector and integer_matrix), and

- basic geometric objects (classes dd_rat_point, dd_rat_vector, dd_rat_direction, dd_rat_hyperplane, dd_rat_segment, dd_rat_ray, dd_rat_line, dd_rat_sphere, and dd_aff_transformation).

On top of the kernel we implemented some classical computation tasks in higher-dimensional CG like convex hulls and Delaunay triangulations. To make the kernel a toolbox for a wide user community and to give the whole project some pedagogical value we aimed for the following.

Ease of use-we aimed for a natural and intuitive interface as far as construction of objects, the conversion between objects, and the interaction of the classes and the operations are concerned. The naming scheme tries to achieve a compromise between mnemonics and word length. We followed a clean, complete and adaptable documentation scheme which provides all necessary information for the user of the kernel and at the same time is integrated into the implementation to enforce consistency between implementation and documentation. The information provided by this manual production toolset consists of prototype information, semantic preconditions, helpful implementation details and runtime information.

Functionality - we tried to provide a comprehensive functionality of the objects while avoiding interface bloating. The identification of the set of primitives for higher level geometric applications was partly a dynamic process influenced by application design (see Section 7).

Layered design-we designed the kernel in a layered fashion for several reasons. First, the functionality of the lower levels is interesting in its own right. In particular, it can be used to realize additional geometric primitives. Second, the fact that the linear algebra layer provides extensive testing and checking routines considerably simplified the development of the geometry layer.

Efficiency - the code is designed to be as fast as possible respecting our primary goal: to develop modular, reusable and maintainable components which are not prone to arithmetic shortcomings like rounding errors. To optimize the runtime behaviour we use the LEDA memory management and a handle-rep scheme to improve memory consumption and to allow identity tests on objects.

The design of our kernel was mainly influenced by three sources: the experiences with the twodimensional LEDA geometry kernel [16], our experiences with an experimental higher-dimensional kernel [17], and discussions with the group developing the CGAL-kernel [9].

In this paper we want to give an overview of the kernel and the applications on top of it. We refer the reader to our web-site [10] for the complete set of manual pages and for the complete documentation of the kernel and the application packet.

In the following sections we describe the three software layers of the kernel and the application layer, give implementation details, and report about experience with the two software packets.

\section{Arithmetic}

The bottom layer of our kernel is exact integral computing. We use the LEDA datatypes integer and rational. Any other bignum package providing the required functionality could be used instead.

The LEDA type integer realizes the mathematical type integer. The arithmetic operations,,$+- *$, $/,+=,-=,-$ (unary),,++-- , the modulus operation $(\%, \%=)$, bitwise AND $(\&, \&=)$, bitwise OR $(||=$,$) , the complement operator (\sim)$, the shift operators $(\ll, \gg)$, the comparison operators $<$, 
Table 1

Runtime comparison of integer arithmetic

\begin{tabular}{|c|c|c|c|c|c|c|c|c|c|}
\hline \multirow[t]{2}{*}{ \#Bits } & \multirow[t]{2}{*}{ \#Ops } & \multicolumn{4}{|r|}{ LEDA } & \multicolumn{4}{|c|}{ GNU in seconds } \\
\hline & & \multicolumn{2}{|c|}{+} & \multicolumn{2}{|c|}{-} & \multicolumn{2}{|c|}{$*$} & \multicolumn{2}{|c|}{1} \\
\hline 32 & $10^{6}$ & 1.1 & 5.1 & 1.3 & 5.5 & 1.6 & 7.5 & 4.7 & 26.6 \\
\hline 64 & $10^{6}$ & 1.2 & 5.0 & 1.3 & 5.4 & 4.2 & 10.9 & 24.8 & 36.6 \\
\hline 500 & $10^{6}$ & 1.8 & 7.3 & 1.86 & 7.8 & 158.8 & 272.7 & 225.7 & 375.3 \\
\hline 1000 & $10^{6}$ & 2.7 & 10.4 & 2.8 & 13.4 & 591.6 & 1063.4 & 736.5 & 1281.3 \\
\hline
\end{tabular}

$\leqslant,>, \geqslant,==, !=$, and the stream operators are all available. These operations never overflow and always yield the exact result. Of course, they may run out of memory.

Integers are essentially implemented by a vector of unsigned longs. The sign and the size are stored in extra variables. Some time critical functions are implemented in SPARC assembler code. The running time of addition is linear. In the multiplication Karazuba-Offman method [11] is used. Thus the running time is $\mathrm{O}\left(L^{\log 3}\right)$, where $L$ is the length of the operands.

A LEDA rational is essentially a pair of integers. The arithmetic operations $+,-, *, /,+=,-=$, $*=, /=,-$ (unary),,++-- are available on rationals. In addition, there are functions to extract the numerator and denominator, to cancel out the greatest common divisor of numerator and denominator, to compute squares and powers, to round rationals to integers, and many others. LEDA's rational numbers are not necessarily normalized, i.e., numerator and denominator of a rational number may have a common factor. A call p.normalize( ) normalizes $p$. This involves a gcd-computation to find the common factor $m$ in numerator and denominator and two divisions to remove the $m$. Since normalization is a fairly costly process we do not do it automatically.

We have run some runtime tests to compare the LEDA integers with the GNU Integers included in GNU's C++ library. The tests have been executed on an Ultrasparc 140. We tested the four basic operations $+,-, *, /$ iteratively on a table of $k$-bit random numbers. The results are shown in Table 1. A more comprehensive test description can be found in [20].

\section{Linear algebra}

The second layer of our kernel provides the standard operations of linear algebra encapsulated in the two classes integer_vector and integer_matrix. These are vectors and matrices with integer entries (= entries of type integer). The possible operations on matrices and vectors include the following.

Construction mechanisms-we can construct $m$-vectors, $m \times n$-matrices from a tuple of equidimensional vectors, identity matrices etc.

Data access operations - we can access the integral and rational components, the dimensions of matrices and vectors, and the rows and columns of matrices.

Arithmetic operations-we provide the inner product, scalar multiplication to vectors and matrices, and vector- and matrix-addition and -multiplication.

Operations based on solving a linear system-we can determine the solution of a linear system $A x=b$, calculate the determinant, rank, inverse, and independent columns of a matrix. 


\section{Deflnition}

An instance of data type integer_matrix is a matrix of variables of type integer, the so called ring type. The arithmetic type integer is required to behave like integers in the mathematical sense ...

\section{Creation}

integer_matrix $M$ (int $n$, int $m$ ); creates an instance $M$ of type integer_matrix of dimension $n \times m$.

integer_matrix $M($ array< integer_vector $>A)$;

creates an instance $M$ of type integer_matrix. Let $A$ be an array of $m$ column - vectors of common dimension $n$. $M$ is initialized to an $n \times m$ matrix with the columns as specified by $A$.

\section{Operations}

\begin{tabular}{|c|c|c|}
\hline int & $M \cdot \operatorname{dim} 1()$ & returns $n$, the number of rows of $M$. \\
\hline integer_vector\& & $M$.row (int $i)$ & $\begin{array}{l}\text { returns the } i \text {-th row of } M \text { (an } m \text { - vector). } \\
\text { Precondition: } 0 \leq i \leq n-1\end{array}$ \\
\hline integer\& & $M($ int $i$, int $j)$ & $\begin{array}{l}\text { returns } M_{i, j} \\
\text { Precondition: } 0 \leq i \leq n-1 \text { and } 0 \leq j \leq m-1\end{array}$ \\
\hline integer_matrix & $M+M 1$ & $\begin{array}{l}\text { Addition. } \\
\text { Precondition: } \\
M \cdot \operatorname{dim} 1()=M 1 \cdot \operatorname{dim} 1() \text { and } M \cdot \operatorname{dim} 2()= \\
M 1 . \operatorname{dim} 2() .\end{array}$ \\
\hline bool & $\begin{array}{r}\text { inverse(integer_matrix } M, \text { intege } \\
\text { determines whether } M \text { h } \\
(1 / D) \cdot \text { inverse or a vecto }\end{array}$ & $\begin{array}{l}\text { atrix \& inverse, integer\& } D \text {, integer_vector\& } c \text { ) } \\
\text { inverse. It also computes either the inverse as } \\
\text { uch that } c^{T} \cdot M=0 \text {. }\end{array}$ \\
\hline bool & $\begin{array}{r}\text { linear_solver( integer_matrix } M, \\
\text { integer_matrix \& } s p \\
\text { determines the complete } \\
\text { system is unsolvable then } \\
\text { then }(1 / D) x \text { is a solution } \\
\text { mal set of linearly indepe } \\
\text { system. } \\
\text { Precondition: } M \cdot \operatorname{dim} 1()\end{array}$ & $\begin{array}{l}\text { er_vector } b, \text { integer_vector\& } x \text {, integer\& } D, \\
\text { ng_vectors, integer_vector\& } c) \\
\text { ion space of the linear system } M \cdot x=b \text {. If the } \\
M=0 \text { and } c^{T} \cdot b \neq 0 \text {. If the system is solvable } \\
d \text { the columns of spanning_vectors are a maxi- } \\
\text { it solutions to the corresponding homogeneous } \\
\lim () .\end{array}$ \\
\hline
\end{tabular}

\section{Implementation}

The datatype integer_matrix is implemented by two-dimensional arrays of variables of type integer. Operations determinant, inverse, linear_solver, and rank take time $O\left(n^{3}\right)$, column takes time $O(n)$, row, $\operatorname{dim} 1, \operatorname{dim} 2$, take constant time, and all other operations take time $O(n m)$. The space requirement is $O(n m)$.

Fig. 1. The manual page of class integer_matrix, an excerpt. 
Table 2

Runtime comparison of linear algebra modules

\begin{tabular}{rrcc}
\hline $\operatorname{dim}$ & LEDA & Maple V R3 & Mathematica 2.0 \\
\hline 20 & $1.3 \mathrm{~s}$ & $15.8 \mathrm{~s}$ & $31.6 \mathrm{~s}$ \\
30 & $7.6 \mathrm{~s}$ & $92.4 \mathrm{~s}$ & $211.3 \mathrm{~s}$ \\
40 & $28.4 \mathrm{~s}$ & $363.7 \mathrm{~s}$ & $840.3 \mathrm{~s}$ \\
100 & $32 \mathrm{~min}$ & $>1 \mathrm{~h}$ & $>1 \mathrm{~h}$ \\
\hline
\end{tabular}

The core operation of the last category is a Gaussian elimination scheme for a non-homogeneous linear system $A x=b$ as described by Edmonds [8]. For a recent reference see the books of Schrijver [19, Part I] or Yap [21, Lecture X]. Basically we transform the original matrix into a diagonalized matrix of integral entries which encodes the numerators of the solution vector and accumulate in parallel a common denominator of the numerator entries.

Fig. 1 shows an excerpt of the manual page of class integer matrix. The same style is used for all other types of the kernel. Each implemented class is documented by four major sections Definition, Creation, Operations and Implementation. The first part gives an overview of the class specification like intended usage of the class and implementation features which influence this usage like exact arithmetic and proof features. The second and third part describe the user accessible operations of the class like constructors, member operations, and functions which work on the class. The description of each operation gives the semantics of it, describes the result transfer by return values and reference parameters, and states preconditions for the usage of the operation. The fourth part of our manual page completes the description by helpful implementation details and runtime and space bounds.

The cited description of the friend function linear solver shows nicely the proof feature of our core operation. Either a solution $x$ is calculated which can be easily checked by substitution into the linear system $M x=b$ or a vector $c$ is provided which proves the unsolvability of the system. Of course there is also a selftest incorporated in the code which can be switched on by a compilation flag and thus the testing can be done permanently.

In a second runtime test we compared the LEDA linear algebra module with commercial math packages like Maple $V$ and Mathematica 2.0. We solved randomly generated non-homogeneous linear systems with $\mathrm{dim}$ rows and columns and 32 bit entries. The tests were executed on a Sun Sparc 4 with $40 \mathrm{MHz}$. The results are shown in Table 2.

\section{Geometric classes}

As mentioned above we provide the geometric classes dd_rat_point, dd_rat_vector, dd_rat_direction, dd_rat_hyperplane, dd_rat_segment, dd_rat_ray, dd_rat_line, dd_rat_sphere and dd_aff_transformation.

We first give a motivation for our interface design and review briefly the basics of analytical geometry. We use $d$ to denote the dimension of the ambient space and assume that our space is equipped with a standard Cartesian coordinate system. The basic object within this space is a point $p$, which we identify with its Cartesian coordinate vector $p=\left(p_{0}, \ldots, p_{d-1}\right)$, where the $p_{i}, 0 \leqslant i<d$, are rational numbers. We store a dd_rat_point by homogeneous coordinates $\left(h_{0}, \ldots, h_{d}\right)$ where $p_{i}=h_{i} / h_{d}$ 
for all $i, 0 \leqslant i<d$, and the $h_{i}$ 's are integer (LEDA type integer). The homogenizing coordinate $h_{d}$ is always positive.

Points, vectors and directions are closely related but nevertheless clearly distinct types. In order to work out the relationship, it is useful to identify a point with an arrow extending from the origin (= an arbitrary but fixed point) to the point. In this view a point is an arrow attached to the origin. A vector is an arrow that is allowed to float freely in space, more precisely, a vector is an equivalence class of arrows where two arrows are equivalent if one can be moved into the other by a translation of space. Points and vectors can be combined by some arithmetical operations. For two points $p$ and $q$ the difference $p-q$ is a vector ( $=$ the equivalence class of arrows containing the arrow extending from $q$ to $p$ ) and for a point $p$ and a vector $v, p+v$ is a point.

All operations of linear algebra apply to vectors, i.e., vectors can be stretched and shrunk (by multiplication with a scalar) and inner and cross product applies to them. On the other hand, geometric tests like affine collinearity or orientation only apply to points. Note that we distinguish the vector

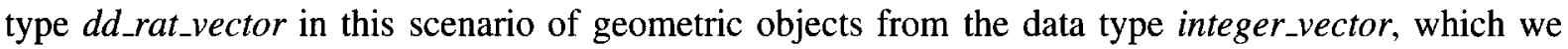
use to formulate calculations in our arithmetic linear algebra layer. We cannot identify both because their role and thereby their functionality within their respective code module is quite different and we don't want to have this mixed up.

A direction is also an equivalence class of arrows, where two arrows are equivalent if one can be moved into the other by a translation of space followed by stretching or shrinking. Alternatively, we may view a direction as a point on the unit sphere. In two-dimensional space directions correspond to angles. As in the case of dd_rat_point we store dd_rat_vector and dd_rat_direction, respectively, as a homogeneous tuple of integers with positive homogenizing component.

The common one-dimensional straight-line objects in $d$-space like lines, rays and segments (which we allow to be trivial) are implemented in the classes $d d_{-}$rat_line, dd_rat_ray and dd_rat_segment and determined by a pair of points.

With respect to the user interface we can group together points, vectors, directions, and on the other hand segments, rays and lines. For the first group there are common operations to access Cartesian and homogeneous coordinates. Conversions within the first group can be made by explicit operations. For the second group there are similar operations to access the coordinates of the determining pair of points.

Oriented hyperplanes in the class dd_rat_hyperplane can be used to model halfspaces and affine hulls of $(d-1)$-dimensional point sets. They are internally stored as a $(d+1)$-tuple of integer

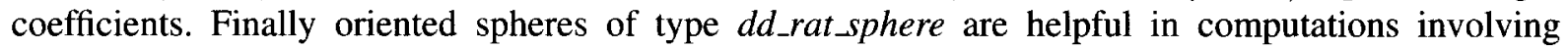
proximity calculations like Voronoi diagrams or Delaunay triangulations. They are stored as a tuple of $d+1$ dd_rat_points.

For all of our basic geometric types we have affine transformations, which can be used by a call of a common member operation which gets an dd_aff_transformation-instance as an argument and delivers a transformed object.

All object classes mentioned use a common handle-rep scheme which is already used in many modules of LEDA. We distinguish between a front-end object which is created by the constructor and a storage object of concrete geometric information which is referenced from the front-end object. The advantages of this scheme emerge in case of frequent copy construction and assignment where only references have to be redirected and no geometric information has to be copied. For large objects this lessens memory consumption and allows us to improve equality checks by testing the reference 


\section{Definition}

An instance of data type dd_rat_point is a point with homogeneous coordinates in an arbitrary dimensional space...

\section{Creation}

dd_rat_point $p($ int $d=2)$;

introduces a variable $p$ of type dd_rat_point in $d$-dimensional space.

dd_rat_point $p($ integer_vector $c$, integer $D)$;

introduces a variable $p$ of type $d_{d}$ rat_point initialized to the point with homogeneous coordinates $\left( \pm c_{0}, \ldots, \pm c_{d-1}, \pm D\right)$, where $d$ is the dimension of $c$ and the sign chosen is the sign of $D$.

Precondition: $D$ is non-zero.

\section{Operations}

int

rational

integer

dd_rat_vector

dd_rat_point

$$
p \cdot \operatorname{dim}()
$$

$p \cdot \operatorname{coord}($ int $i)$

$p \cdot h \operatorname{coord}($ int $i)$

$p-q$

$p+d d_{-}$rat_vector $v$ returns the dimension of $p$.

returns the $i$ th Cartesian coordinate of $p$.

returns the $i$ th homogeneous coordinate of $p$.

returns $p-q$.

Precondition: $p \cdot \operatorname{dim}()==q \cdot \operatorname{dim}()$.

returns $p+v$.

Precondition: $p \cdot \operatorname{dim}()==v \cdot \operatorname{dim}()$.

int orientation (array< dd_rat_point $>A)$

determines the orientation of the points in $A$, where $A$ consists of $d+1$ points in $d$ space. This is the sign of the determinant

$$
\left|\begin{array}{cccc}
1 & 1 & 1 & 1 \\
A[0] & A[1] & \ldots & A[d]
\end{array}\right|
$$

where $A[i]$ denotes the Cartesian coordinate vector of the $i$ th point in $A$.

Precondition: A.size( $)==d+1$ and $A[i] \cdot \operatorname{dim}()==d \forall 0 \leq i \leq d$.

bool contained_in_affine hull (array< dd_rat_point $>A, d d$ _rat_point $x)$

determines whether $x$ is contained in the affine hull of the points in $A$.

\section{Implementation}

Points are implemented by arrays of integer as an item type. All operations like ...

Fig. 2. The manual page of class dd_rat point, an excerpt. 
addresses before a comparison of geometric coordinate information. As in the case of our linear algebra module we use LEDA's improved memory management module which gives us a certain speed-up compared to the standard $\mathrm{C}++$ allocation scheme.

We now take a closer look at two example manual pages, by which we elaborate further on the features of our data types. Fig. 2 shows an excerpt from the point class manual page.

The default dimension of all objects is 2 . This means that a call to the standard constructor $d d_{\text {_rat_point }}$ ) delivers an instance of type dd_rat_point for planar geometry. A point in $d$-dimensional space is constructed by $p$ (integer_vector $c$, integer $D)$ or $p($ integer_vector $c)$. In addition, there are standard initialization operations which allow comfortable creation of objects for the dimensions 2 and 3, which are not shown here. The data access operations allow access to the dimension and to Cartesian and homogeneous coordinates. Operator overloading allows the intuitive calculation of $d d_{-}$rat_point difference, which results in a dd_rat_vector and the translation of $d d_{\text {_rat }}$ points by adding a dd_rat_vector. The orientation predicate and other affine operations use LEDA arrays as a container type for a tuple of points.

The basic operations provided on straight line objects are mainly position checks of points with respect to the objects, like contains ( ) and intersection calculation between all higher dimensional objects like segments, rays, lines and hyperplanes.

To manipulate all the basic objects we implemented affine transformations in form of a class dd_aff_transformation. Objects of this type are basically transformation matrices which can be applied to the objects by the common member function transform ( ). We provide basic construction of such transformations like translation, scaling, rotations of a $2 \mathrm{D}$-subspace spanned by base vectors. As general planar rotations specified by angles are not representable by rational coefficients we offer a mechanism to construct rational approximated rotation instances calculated with respect to a given direction (angle) and an error bound as described in [4]. The primitive affine transformations can finally be combined. The usage of this class is presented in Fig. 3.

\section{Applications: convex hulls and Delaunay triangulations}

The convex hull and the Delaunay triangulation problem are traditionally specified as functions, i.e., given a set of points compute their convex hull or their Delaunay triangulation in some representation. We specify both problems as data types that support insertions and a large variety of query operations. In the case of convex hulls we support navigation through the interior and the boundary of the hull and we support membership and visibility queries. In the case of Delaunay triangulations we support navigation through the triangulation, we support locate ${ }^{1}$ and nearest neighbor queries, and we support range queries with spheres and simplices. For two-dimensional convex hulls and Delaunay triangulations we also support an interface to the LEDA graph and window classes $[14,16]$. In this way one can, for example, construct two-dimensional nearest and furthest site Voronoi diagrams and minimum spanning trees, display hulls and Delaunay triangulations.

The next two sections present parts of the specifications of convex hulls and Delaunay triangulations, respectively.

\footnotetext{
${ }^{1}$ A locate query finds the simplex of the triangulation containing the query point.
} 


\section{Definition}

An instance of the data type dd_aff_transformation is an affine transformation of $d$-dimensional space. It is specified by a square integer matrix $M$ of dimension $d+1$. All entries in the last row of $M$ except the diagonal entry must be zero; the diagonal entry must be non-zero. A point $p$ with homogeneous coordinates $(p[0], \ldots, p[d])$ can be transformed into the point p.transform $(A)$, where $A$ is an affine transformation created from $M$ by the constructors below.

\section{Creation}

dd_aff_transformation $t($ int $d=2)$;

introduces a transformation in $d$-dimensional space.

\section{Operations}

dd_aff_transformation dd_aff_transformation:: translation(dd_rat_vector vec)

returns a translation corresponding to the translation vector vec.

dd_aff_transformation dd_aff_transformation::scaling(integer num, integer den, int $d=2$ ) returns a scaling by a scale factor num/den.

dd_aff_transformation dd_aff_transformation:: planar_rotation(dd_rat_direction dir, integer num, integer den, int $e 1=0$, int $e 2=1$, int $d=2$ )

returns a planar rotation within the plane spanned by the base vectors $b_{e 1}$ and $b_{e 2}$ in $d$-space. The rotations parameters are given by the 2-dimensional direction dir, such that the difference between the sines and cosines of the rotation given by dir and the approximation rotation are at most $n u m /$ den each.

Precondition: $\operatorname{dir} \cdot \operatorname{dim}()==2$ and $n u m<\operatorname{den}$ is positive and $0 \leq$ $e_{1}<e_{2}<d$

dd_aff_transformation $t 2 * t 1$

composition of transformations. Note that transformations are not necessarily commutative. $t 2 * t 1$ is the transformation which transforms first by $t 1$ and then by $t 2$.

\section{Implementation}

Affine Transformations are implemented by matrices of integers as an item type. All operations like creation, initialization, input and output on a transformation $t$ take time $O\left(t \operatorname{dim}()^{2}\right) \cdot \operatorname{dim}()$ takes constant time. The operations for inversion and composition have the cubic costs of the used matrix operations. The space requirement is $O\left(\operatorname{td} \operatorname{dim}()^{2}\right)$.

Fig. 3. The manual page of class dd_aff_transformation, an excerpt. 


\subsection{Convex hulls}

An instance $C$ of type chull is the convex hull of a multi-set $S$ of points in $d$-dimensional space. We call $S$ the underlying point set and $d$ or $\operatorname{dim}$ the dimension of the underlying space. We use $d c u r$ or dcurrent to denote the affine dimension of $S$. The data type supports incremental construction of hulls.

The closure of the hull is maintained as a simplicial complex, i.e., as a collection of simplices where the intersection of any two is a face of both. ${ }^{2}$ In the sequel we reserve the word simplex for the simplices of dimension dcur. For each simplex there is an item of type ch_simplex and for each vertex there is an item of type ch_vertex. Each simplex has $1+d c u r$ vertices indexed from 0 to dcur; for a simplex $s$ and an index $i, C$.vertex $(s, i)$ returns the $i$ th vertex of $s$. For any simplex $s$ and any index $i$ of $s$ there may or may not be a simplex $t$ opposite to the $i$ th vertex of $s$. The function C.opposite_simplex $(s, i)$ returns $t$ if it exists and returns nil otherwise. If $t$ exists then $s$ and $t$ share $d c u r$ vertices, namely all but the vertex with index $i$ of $s$ and the vertex with index C.index_of_vertex_in_opposite_simplex $(s, i)$ of $t$. Assume that $t$ exists and let $j=C$.index_of_vertex_in_opposite_simplex $(s, i)$. Then $s=C$.opposite_simplex $(t, j)$ and $i=$ C.index_of_vertex_in_opposite_simplex $(t, j)$.

\subsection{Delaunay triangulations}

An instance $D T$ of type $d d$ delaunay is the nearest and furthest site Delaunay triangulation of a set $S$ of points in some $d$-dimensional space. We call $S$ the underlying point set and $d$ or $d i m$ the dimension of the underlying space. We use dcur or dcurrent to denote the affine dimension of $S$. The data type supports incremental construction of Delaunay triangulations and various kinds of query operations (in particular, nearest and furthest neighbor queries and range queries with spheres and simplices).

A Delaunay triangulation is a simplicial complex. All simplices in the Delaunay triangulation have dimension $d c u r$. In the nearest site Delaunay triangulation the circumsphere of any simplex in the triangulation contains no point of $S$ in its interior. In the furthest site Delaunay triangulation the circumsphere of any simplex contains no point of $S$ in its exterior. If the points in $S$ are co-circular then any triangulation of $S$ is a nearest as well as a furthest site Delaunay triangulation of $S$. If the points in $S$ are not co-circular then no simplex can be a simplex of both triangulations. Accordingly, we view $D T$ as either one or two collection of simplices. If the points in $S$ are co-circular there is just one collection: the set of simplices of some triangulation. If the points in $S$ are not co-circular there are two collections. One collection consists of the simplices of a nearest site Delaunay triangulation and the other collection consists of the simplices of a furthest site Delaunay triangulation.

For each simplex there is an item of type dtsimplex and for each vertex of the triangulation there is an item of type dt_vertex. Each simplex has $1+d c u r$ vertices indexed from 0 to dcur. Similar operations as for convex hulls are provided. For any simplex $s$ and any index $i, D T$.vertex_of $(s, i)$ returns the $i$ th vertex of $s$. There may or may not be a simplex $t$ opposite to the vertex of $s$ with index $i$. There are similar adjacency operations as listed for the chull type above. In general, a vertex belongs to many simplices.

\footnotetext{
${ }^{2}$ The empty set is a facet of every simplex.
} 
Any simplex of $D T$ belongs either to the nearest or to the furthest site Delaunay triangulation or both in the degenerate case. The test DT.simplex_of_nearest (dt_simplex $s$ ) returns true if $s$ belongs to the nearest site triangulation and the test DT.simplex_of_furthest (dt_simplex $s$ ) returns true if $s$ belongs to the furthest site triangulation.

\subsection{Further implementation issues}

The implementation of type chull follows [5] and Delaunay triangulations are reduced to convex hulls through the well-known lifting map, see, for example, [6,7]. Based on our kernel a class regl_complex was implemented that can represent so-called regular simplicial complexes. A simplicial complex is called regular if all maximal simplices, i.e., simplices that are not a subsimplex of another simplex of the complex, have the same dimension. The class regl_complex provides operations for navigation through the complex and update operations. The class chull is derived from regl_complex and the class dd_delaunay is derived from chull.

The insertion of a new point $x$ to the convex hull follows a simple scheme. If $x$ implies a dimension jump ( $x$ is not contained in the affine hull of the current convex hull) then the update of $C$ is just an expansion of all simplices of $C$ by a new vertex located in $x$. If $x$ implies no dimension jump then $C$ must only be updated if $x$ is outside the hull and the update concerns all boundary facets visible from $x$. The implementation handles degenerate inputs as described in [3].

The work horse for the query operations on convex hulls and Delaunay triangulations is a method

$$
\begin{gathered}
\text { C.visibility_search(dd_rat_point } \mathrm{x}, \text { list<ch_facet }>\& \text { visible_facets, } \\
\text { int\& location, ch_facet\& f); }
\end{gathered}
$$

that constructs the list of all $x$-visible hull facets in visible facets, returns the position of $x$ with respect to the current hull in location ( -1 for inside, 0 for on the boundary, and +1 for outside) and, if $x$ is contained in the boundary of $C$, returns a facet incident to $x$ in $f$.

The membership query and the visible facets query for hulls are easily realized by this method and the nearest neighbor and the range query for Delaunay triangulations use it in an essential way. The nearest neighbor query for Delaunay triangulations lifts the query point (using the lifting map), then determines all visible facets of the hull, and then selects the best vertex by linear search through their vertices. This method is only efficient in low-dimensional space. The range query with spheres lifts the sphere (using the lifting map) and then finds all vertices of the hull that lie below the resulting hyperplane which is obtained by the lifting map.

We use program checking $[2,15]$ in our implementation. In particular:

- the class regl_complex provides a method $R C$.check_topology( ) that partially checks whether $R C$ is an abstract simplicial complex, ${ }^{3}$ and a method $R C$.is_Delaunay (kind) that checks whether $R C$ is a nearest $($ kind $=$ nearest) or furthest $($ kind $=$ furthest ) site Delaunay triangulation of its vertex set; - the class chull provides a method C.check( ) that verifies convex hulls as described in [15];

\footnotetext{
${ }^{3}$ The method checks whether the neighborhood relationship on simplices is symmetric, whether all vertices of a simplex are distinct, and whether two neighboring simplices share all but one of their vertices. It does not check whether simplices that share all but one of their vertices are actually neighbors in the complex.
} 
- we have algorithms that check whether a graph is a nearest or furthest site Delaunay diagram of its vertex set, whether a graph is a triangulation of its vertex set, and whether a graph is a nearest or furthest site Voronoi diagram.

The representation of convex hulls and Delaunay triangulations in data types chull and dd_delaunay is simplex-based, i.e., simplices are the main objects and lower dimensional faces are only implicitly represented. In two-dimensional space there is an alternative representation which makes the vertices and edges the primary objects and represents simplices (= triangles) implicitly as faces of a planar graph. This is the representation used for two-dimensional Delaunay triangulations (type delaunay) in LEDA. If $D T$ has type dd_delaunay, DTG has type $G R A P H<P O I N T$, int $>,{ }^{4}$ and $k i n d$ is one of nearest or furthest, then

$$
\text { DT.graphrep(DTG, kind); }
$$

constructs the graph representation of $D T$ in $D T G$. All LEDA graph algorithms can then be applied to $D T G$. For example,

compute_voronoi(DTG, VD, kind);

will construct the graph representation of the Voronoi diagram in $V D$, and

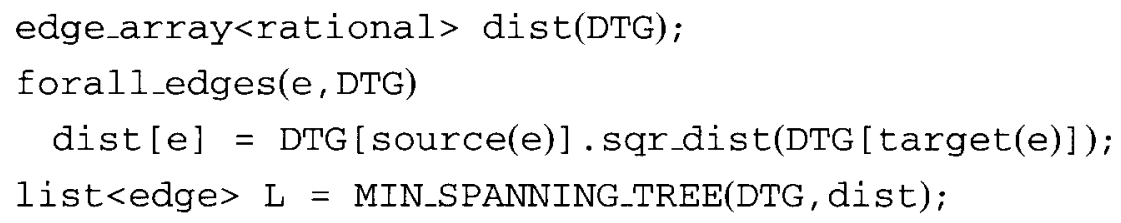

will construct in $L$ the set of edges comprising a minimum spanning tree of DTG.

\section{Documentation}

The full documentation of the kernel consists of about 240 pages [10] and the documentation of the application layer comprises about 100 pages [12].

Fig. 4 shows the implementation of the orientation-predicate of the class $d d$ rat point and one member operation of class chull. For each class the documentation and the implementation are collected in a noweb-file. See [18] for an introduction to noweb. Different tools are used to give different views of the noweb-file: the noweb tool notangle extracts the code, i.e., the view needed by the $\mathrm{C}++$ compiler, and the LEDA tools Lman and Ldoc give the manual view and the documentation view, respectively. See [13] for an introduction to this tools.

The figure also illustrates the vertical interaction between the software layers. In case of the orientation-predicate the determinant calculation is done by determinant( ) provided as a friend of integer.matrix. Most affine operations on point tuples map to the solution of a corresponding linear system calculated by our linear algebra layer. Notice that precondition checks are formulated as preprocessor macros which can be switched off by the flag -DLEDA_CHECKING_OFF.

\footnotetext{
${ }^{4}$ In LEDA, GRAPH<POINT, int $>$ is the type of graphs where each node has an associated information of type POINT and each edge has an associated information of type int; the edge information is not used in this code.
} 


\section{Orientation}

$\left\langle d_{\text {rrat_point. }}\right\rangle \equiv$

int orientation(const array<rat_point $>A$ );

Semantics: determines the orientation of the points in $A$, where $A$ consists of $d+1$ points in $d$-space. This is the sign of the determinant

$$
\left|\begin{array}{cccc}
1 & 1 & 1 & 1 \\
A[0] & A[1] & \ldots & A[d]
\end{array}\right|
$$

where $A[i]$ denotes the Cartesian coordinate vector of the $i$ th point in $A$.

We are given an array $A$ of $d+1$ points in $d$-space and compute their orientation. Multiplying the $i$ th column of the above matrix by the homogenizing coordinate of $A[i]$ leaves the sign of the determinant unchanged. We set up this matrix and return its determinant. Actually, it is more convenient to transpose it and to make the first row the last. This changes the sign if the number of rows is even, i.e., if $d$ is odd.

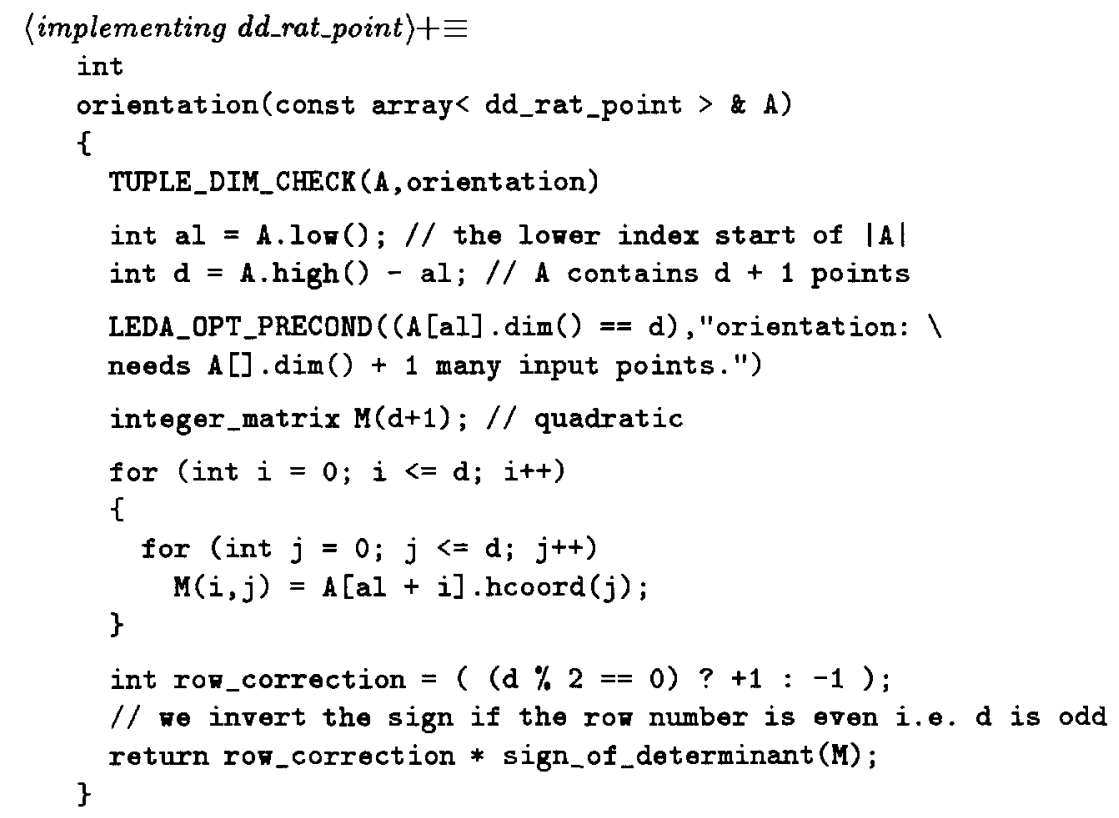

Fig. 4. The implementation style.

\section{Experimental experiences}

Both programs rely heavily on the fact that the kernel is exact. For example, the insertion routine for convex hulls distinguishes cases according to whether the newly inserted point lies in the affine hull of the points already present or not, and the checking programs would hardly make sense without exact primitives. 
In the early stages of program development the checking feature of the kernel was particularly useful. For example, the convex hull program needs to compute the hyperplane defined by a set of points. This can be done by solving a linear system. In the first version of the program we set up the wrong linear system. It was very useful that the linear system solver gives a proof of unsolvability and does not just claim unsolvability. This located the error fairly quickly.

We have used classes chull and dd_delaunay on problems up to dimension 10 . We have also compared it to the qhull-program of Barber et al. [1] and the hull-program of Clarkson. The first method computes approximate convex hulls and the latter method computes exact hulls but works only for a limited (albeit large) range of coordinate values. Both methods are significantly faster than ours. This is mostly due to their use of floating point arithmetic. Neither of the algorithms provides the rich functionality that we provide.

\section{Availability and extensions}

The whole kernel has been used internally since June 96 and is available in form of an extension package to the LEDA software library. The complete specification of the listed data types and the code projects can be obtained via WWW from the LEDA home page http://www. mpi-sb.mpg . de/LEDA.

In cooperation with the CGAL-project the code base was extended with number type templatization. This allows runtime comparisons depending on the plugged in arithmetic components. We want to evaluate the use of modular integer data types within the linear algebra packet as well as in different geometric application scenarios. An adapted version of the templatized code will become part of the CGAL-kernel.

\section{Conclusions}

We described the layered design of a kernel for higher dimensional computational geometry and gave two examples for applications. The primary goal was to develop a useful base for geometric application design including checkable correctness, efficiency based on existing software library concepts and a clear and comprehensive documentation scheme.

\section{References}

[1] C. Barber, D. Dobkin, H. Hudhanpaa, The quickhull program for convex hulls, ACM Trans. Math. Software 22 (1996) 469-483.

[2] M. Blum, M. Luby, R. Rubinfeld, Self-testing/correcting with applications to numerical problems, in: Proc. 22nd Annual ACM Symp. on Theory of Computing, 1990, pp. 73-83.

[3] C. Burnikel, K. Mehlhorn, S. Schirra, On degeneracy in geometric computations, in: Proc. SODA '94, 1994, pp. 16-23.

[4] J. Canny, B.R. Donald, E.K. Ressler, A rational rotation method for robust geometric algorithms, in: Proc. of the 8th ACM Symposium on Computational Geometry, 1992, pp. 251-260.

[5] K.L. Clarkson, K. Mehlhorn, R. Seidel, Four results on randomized incremental constructions, Computational Geometry: Theory and Applications 3 (1993) 185-212. 
[6] H. Edelsbrunner, Algorithms in Combinatorial Geometry, Springer, Berlin, 1987.

[7] H. Edelsbrunner, R. Seidel, Voronoi diagrams and arrangements, in: J. O'Rourke (Ed.), Proceedings of the Symposium on Computational Geometry, Baltimore, MD, June 1985, ACM Press, New York, pp. 251-262.

[8] J. Edmonds, Systems of distinct representatives and linear algebra, J. Res. Nat. Bureau Standards 71(B) (1967) 241-245.

[9] A. Fabri, G.-J. Giezeman, L. Kettner, S. Schirra, S. Schönherr, The CGAL kernel: A basis for geometric computation, in: M.C. Lin, D. Manocha (Eds.), Applied Computational Geometry: Towards Geometric Engineering: Workshop (FCRC-96; WACG-96), Philadelphia, PA, USA, 27-28 May 1996; selected papers, in: Lecture Notes in Computer Science, Vol. 1148, Springer, Berlin, 1996, pp. 191-202.

[10] Lep distribution: http: / / www .mpi-sb.mpg.de/LEDA/www/leps/dd_geokernel . html.

[11] D.E. Knuth, The Art of Computer Programming, Vol. 2, Seminumerical Algorithms, Addison-Wesley, Reading, MA, 1969.

[12] K. Mehlhorns home page: http://www.mpi-sb.mpg.de/ mehlhorn/Programs.html.

[13] LEDA book preview: http://www.mpi-sb.mpg. de/ mehlhorn/LEDAbook. html.

[14] K. Mehlhorn, S. Näher, LEDA, a platform for combinatorial and geometric computing, Commun. ACM 38 (1995) 96-102.

[15] K. Mehlhorn, S. Näher, T. Schilz, S. Schirra, M. Seel, R. Seidel, C. Uhrig, Checking geometric programs or verification of geometric structures, in: Proc. of the 12th Annual Symposium on Computational Geometry, 1996, pp. 159-165.

[16] K. Mehlhorn, S. Näher, C. Uhrig, The LEDA User Manual, 1995.

[17] M. Müller, J. Ziegler, An implementation of a convex hull algorithm, Technical Report MPI-I-94-105, Max-Planck-Institut für Informatik, Saarbrücken, 1994.

[18] Noweb home page: http://www. cs.virginia.edu/ nr/noweb/.

[19] A. Schrijver, Theory of Linear and Integer Programming, Wiley, Chichester, 1986.

[20] M. Seel, A runtime test of integer arithmetic and linear algebra in LEDA, Research Report MPI-I-96-1-033, Max-Planck-Institut für Informatik, Saarbrücken, 1996.

[21] C.K. Yap, Fundamental Problems in Algorithmic Algebra, Princeton University Press, Princeton, NJ, 1997. 